\title{
Cognitive Bias in the COVID-19 Pandemic
}

\author{
Christina N. DiMaria ${ }^{1}$, Byeori Lee ${ }^{1}$, Robert Fischer ${ }^{2}$, Glenn Eiger ${ }^{1}$ \\ 1. Internal Medicine, Einstein Medical Center, Philadelphia, USA 2. Infectious Disease, Einstein Medical Center, \\ Philadelphia, USA
}

Corresponding author: Christina N. DiMaria, dimariac@einstein.edu

\begin{abstract}
Cognitive bias plays a significant role in medical errors. In the pandemic of corona virus disease-19 (COVID19), recognizing and creating strategies to minimize these biases is crucial to optimize medical care for our patients. In this article we present a case of a 68-year-old male with decreased appetite, subjective fears, dry cough, and confusion. The report illustrates the concept of cognitive bias during a pandemic and discusses strategies to ameliorate them.
\end{abstract}

Categories: Internal Medicine, Infectious Disease, Epidemiology/Public Health Keywords: cognitive bias, covid, heuristics

\section{Introduction}

Cognitive bias includes a variety of unconscious influences, heuristics, and behaviors which aid our decision making [1]. These shortcuts can be helpful in clinical decision making, but they can also lead to medical errors. A study by Graber et al. found that $74 \%$ of diagnostic errors in internal medicine practice are related to cognitive factors [2]. Recognizing and creating strategies to minimize these biases is crucial to optimize medical care for our patients. We describe a case of a 68-year-old male with decreased appetite, subjective fears, dry cough, and confusion presenting during the corona virus disease-19 (COVID-19) pandemic. To our knowledge there are no case reports or studies focusing on the role of cognitive biases during a pandemic or strategies to overcome them.

\section{Case Presentation}

A 68-year-old male presented with decreased appetite, fatigue, and confusion for four days. His wife reported worsening disorientation and drowsiness for one day prompting her to bring him to the hospital. Other symptoms included subjective fever and dry cough. He lived at home with his wife and grandchildren and was independent in activities of daily living at baseline. He had hypertension and hyperlipidemia. The patient had no recent travel or sick contacts. His medications included spironolactone, hydrochlorothiazide, losartan, and nifedipine. He had no smoking, alcohol, or drug use history.

Received 06/20/2020 Review began 06/23/2020 Review ended 06/28/2020 Published 07/06/2020

๑) Copyright 2020 DiMaria et al. This is an open access article distributed under the terms of the Creative Commons Attribution License CC-BY 4.0., which permits unrestricted use, distribution, and reproduction in any medium, provided the original author and source are credited.
Vital signs on admission included blood pressure $135 / 77 \mathrm{mmHg}$, oxygen saturation of $97 \%$ on room air, heart rate $80 \mathrm{bpm}$, and temperature 39 degrees Celsius. He had no neck stiffness, no focal neurological deficits, and was alert and oriented to his name. The rest of the physical findings were unremarkable. Chest X-ray showed no acute disease. Noncontrast cranial CT was negative. Serum sodium was $140 \mathrm{mEq} / \mathrm{L}$, potassium 3.8 $\mathrm{mEq} / \mathrm{L}$, creatinine $2.3 \mathrm{mg} / \mathrm{dL}$, WBC 10,000 per microliter with a neutrophil predominance of $80 \%$. A nasal swab was negative for influenza and respiratory syncytial virus (RSV) by polymerase chain reaction (PCR). A nasal swab for COVID-19 PCR was obtained and the patient was admitted to the COVID general medicine floor for "COVID rule out."

While awaiting the COVID test result, the patient was followed clinically. Minimal additional testing was performed. His mental status continued to wax and wane for the next six days, with delirium worsening when his fever was high. No specialty services were consulted. Eventually, COVID-19 testing returned positive and he was treated with symptomatic management.

\section{Discussion}

This case brings into question how we approach medical decision making during the COVID-19 pandemic. Although our patient was eventually diagnosed with COVID-19, there were alternative diagnoses that should have been considered including but not limited to a central nervous system (CNS) infection or vasculitis, seizures, hypercapnia, HIV, or uncontrolled hyperthyroidism.

The primary bias illustrated by our case is premature closure. Premature closure is a cognitive error in which the physician fails to consider reasonable alternatives after an initial diagnosis is suspected [3]. Given the increasing prevalence of COVID-19, it is hard not to focus on this diagnosis when evaluating a patient. After the patient was admitted to the medicine floor, minimal further testing was done as it was thought "prematurely" that the patient had COVID-19. Another cognitive bias demonstrated by this case is 
availability bias -- the mental shortcut that relies on likelihood or frequency of a disease to construct a differential diagnosis [4]. Currently there are 2,027,521 confirmed cases of COVID-19 in the United States [5]. The likelihood of our patient having COVID-19 is high, yet other diseases have not vanished, and other diagnoses are still possible.

On review of the case, the presenting symptoms were vague, yet we focused on dry cough and fever to lead us to a diagnosis of COVID-19. Other symptoms and other physical findings were overlooked. This is an example of anchoring bias.

The final bias observed in our case is framing bias, the influence of how information is presented on medical decision making [6]. This bias occurs at many levels; admission from the ED, medical rounds presentations, handoffs, and signouts. In this case, the general medicine team was called for an admission for "COVID rule out." And this was also the term used for handoffs and signouts. We believe this wording may oversimplify the patient's disease state and focus only on COVID-19.

Yes, in the end, the patient was found to have COVID-19, but what if after six days the test came back negative? Would we have then recognized the biases that affected our medical decision making? Could we have provided better and safer medical care?

Recognition and strategies to prevent cognitive biases, especially during a pandemic, are crucial to optimize medical care for our patients. It would facilitate more efficient diagnosis of non-COVID-19 diseases which would allow for earlier discharges and free up hospital beds. For this reason, we suggest some guiding principles to prevent cognitive bias errors during the COVID-19 pandemic.

1. Three alternative diagnoses in addition to COVID-19

Three alternative diagnoses should be routinely sought for patients with suspected COVID-19 infection. After making sure the patient is screened appropriately for infection control and placed on the proper isolation, it is important to think about common pre-COVID-19 diseases. In addition, COVID-19 positivity does not rule out other diseases. Our patients are complicated and can have multiple diagnoses. Common things will always be common, and we are learning that this is still the case during a pandemic.

\section{Diagnostic Time Out}

After brainstorming differential diagnoses, take a time-out as a team. Ask what else besides COVID-19 can this be? As a team, review the patient's initial symptoms, vital signs, labs and synthesize this with new data or findings found while the patient has been on the floor. During this time out consider how likely COVID19 is for the patient and if potentially a positive test could be a false positive.

\section{Include Specialists}

We, physicians, are a team in this fight against COVID-19. Resources on many levels are stretched thin. For this reason, we think it would be beneficial to involve specialists. Even if they are working via telemedicine, discussing the case with experienced specialists may provide further insight.

\section{Conclusions}

Although cognitive biases are known contributors to cognitive errors, we still are not sure of all the ways they play into medical decision making during this COVID-19 pandemic. By dissecting this case we feel we were able to illustrate the concept of cognitive bias, how it influences our medical care, and suggest a few strategies to overcome them.

\section{Additional Information \\ Disclosures}

Human subjects: Consent was obtained by all participants in this study. Conflicts of interest: In compliance with the ICMJE uniform disclosure form, all authors declare the following: Payment/services info: All authors have declared that no financial support was received from any organization for the submitted work. Financial relationships: All authors have declared that they have no financial relationships at present or within the previous three years with any organizations that might have an interest in the submitted work. Other relationships: All authors have declared that there are no other relationships or activities that could appear to have influenced the submitted work.

\section{References}

1. O'Sullivan ED, Schofield SI: A cognitive forcing tool to mitigate cognitive bias-a randomised control trial . BMC Med Educ. 2019, 19:12. 10.1186/s12909-018-1444-3

2. Graber ML, Franklin N, Gordon R: Diagnostic error in internal medicine. Arch Intern Med. 2005, 165:1493- 


\section{Cureus}

1499. 10.1001/archinte.165.13.1493

3. Kumar B, Kanna B, Kumar S: The pitfalls of premature closure: clinical decision-making in a case of aortic dissection. BMJ Case Rep. 2011, 165:1493-1499. 10.1136/bcr.08.2011.4594

4. Tversky A, Kahneman D: Judgment under uncertainty: heuristics and biases . Science. 1974, 185:1124-1131. 10.1126/science.185.4157.1124

5. COVID-19 Map - Johns Hopkins Coronavirus Resource Center . (2020). Accessed: April 1, 2020: https://coronavirus.jhu.edu/map.html.

6. Gong J, Zhang Y, Feng J, Huang Y, Wei Y, Zhang W: Influence of framing on medical decision making. EXCLI J. 2013, 12:20-29. 10.17877/DE290R-10585 